\title{
Severe Skin Disorders Due to Sorafenib Use After Nivolumab Treatment in Renal Cell Carcinoma Patients
}

\author{
HIROYOSHI KOIDE ${ }^{1}$, SATOSHI NODA ${ }^{1}$, TETSUYA YOSHIDA ${ }^{2}$, SUSUMU KAGEYAMA $^{2}$, KAZUYA TERAMURA $^{3}$, \\ TAKESHI KATO $^{3}$, AKIHIRO KAWAUCHI ${ }^{2}$, NORIKI FUJIMOTO ${ }^{3}$ and TOMOHIRO TERADA ${ }^{1}$ \\ ${ }^{1}$ Department of Pharmacy, Shiga University of Medical Science Hospital, Shiga, Japan; \\ ${ }^{2}$ Department of Urology, Shiga University of Medical Science, Shiga, Japan; \\ ${ }^{3}$ Department of Dermatology, Shiga University of Medical Science, Shiga, Japan
}

\begin{abstract}
Background: We report two cases in which severe skin disorders developed during sorafenib treatment in patients with renal cell carcinoma (RCC) who had previously received nivolumab. Case Report: Case 1: A 50year-old man with RCC received nivolumab as the fifth-line therapy followed by sorafenib as the sixth-line therapy. On day 15 of sorafenib administration, the patient was hospitalized with systemic erythema multiforme, acne-like skin rash, and hand-foot syndrome. Case 2: A 40-year-old man with RCC received nivolumab as the second-line therapy followed by sorafenib as the fifth-line treatment. On day 12 of sorafenib administration, the patient was hospitalized with an acne-like skin rash and hand-foot syndrome. The skin disorders in the two cases improved within 2-3 weeks after sorafenib discontinuation and the start of treatment with topical and oral steroids. Conclusion: When using sorafenib in patients previously treated with nivolumab, close attention should be paid to the onset of serious skin disorders.
\end{abstract}

Molecular-targeted anticancer drugs, which target mutated and/or overexpressed tyrosine kinases [such as vascular endothelial growth factor receptor and epidermal growth factor receptor (EGFR)] in cancer tissues, are among the key drugs for cancer treatment. In recent years, with the advent of immune checkpoint inhibitors (ICIs), which elicit

This article is freely accessible online.

Correspondence to: Hiroyoshi Koide, Ph.D., Department of Pharmacy, Shiga University of Medical Science Hospital, Seta Tsukinowa-cho, Otsu City, Shiga 520-2192, Japan. Tel: +81 775482686, Fax: +81 775482411, e-mail: kd14004@belle.shigamed.ac.jp

Key Words: Sorafenib, nivolumab, skin disorder, immunotherapy, renal cell carcinoma. antitumor effects by activating host immunity [programmed death-1 (PD-1), PD ligand-1, and cytotoxic T-lymphocyteassociated antigen-4], molecular-targeted anticancer drugs and ICIs are being increasingly used simultaneously or sequentially in chemotherapy for various cancers. Moleculartargeted anticancer drugs are considered to have fewer general side effects such as myelosuppression and digestive symptoms than do cytotoxic anticancer drugs. Nevertheless, caution is required while using these drugs because they have side effects such as skin disorders, cardiovascular diseases, and interstitial lung disease. In contrast, ICIs are known to cause immune-related adverse events such as skin disorders, interstitial pneumonia, endocrine disorders, liver disorders, and renal disorders due to immune overreaction. Against this background, ICIs have been reported to have long-lasting effects (1), and may additively or synergistically enhance not only clinical efficacy but also the side effects of molecular-targeted anticancer drugs administered sequentially after ICIs. Indeed, multiple cases of severe toxicities induced by molecular-targeted anticancer drugs, administered after ICIs, have been reported. For instance, interstitial lung disease has been reported in lung cancer patients treated with the EGFR-tyrosine kinase inhibitor osimertinib following treatment with nivolumab, a PD-1 antibody $(2,3)$. In addition, serious skin disorders or hypersensitivity reactions, including Stevens-Johnson syndrome, have been reported in melanoma patients treated with the BRAF inhibitor vemurafenib following ICI therapy $(4,5)$. Thus, in patients treated with molecular-targeted anticancer drugs following ICI therapy, ICIs may reduce the threshold for the development of serious side effects of molecular-targeted anticancer drugs. However, to date, information on the side effects in patients treated with molecular-targeted anticancer agents following ICI therapy remains limited. Here, we report two cases of severe skin disorders caused by sorafenib, a molecular-targeted anticancer agent, in patients with renal cell carcinoma (RCC) who had been previously treated with nivolumab. 


\section{Case Report}

Case 1. A 50-year-old, $72.4 \mathrm{~kg}$ man underwent nephrectomy for left RCC (stage pT3a cN0 cM0, R0) in October 2012. Since lung metastases were detected in July 2013, he received sunitinib, axitinib, everolimus, and pazopanib as the first-, second-, third-, and fourth-line therapies, respectively, until disease progression. In October 2016, he received nivolumab (3 mg/kg, $72.4 \mathrm{~kg}$, every 2 weeks) as a fifth-line therapy. He had stable disease with the best response to nivolumab treatment; however, nivolumab was discontinued in the $11^{\text {th }}$ cycle owing to fever $\left(39^{\circ} \mathrm{C}\right)$ and liver dysfunction [laboratory tests revealed increased levels of aspartate transaminase $(148 \mathrm{U} / \mathrm{l})$, alanine transaminase $(127 \mathrm{U} / 1)$, and $\gamma$-glutamyl transpeptidase $(174 \mathrm{U} / \mathrm{l})]$. No serious skin disorder was observed during treatment with sunitinib, axitinib, everolimus, pazopanib, and nivolumab before the start of sorafenib administration. After disease progression, treatment was switched to sorafenib (800 mg daily) as a sixth-line therapy in April 2017. The interval between the last dose of nivolumab and the initiation of sorafenib was 28 days. At this point, the patient was categorized as having intermediate risk according to the Memorial Sloan Kettering Cancer Center (MSKCC) risk criteria and was in a good general condition with an Eastern Cooperative Oncology Group performance status (ECOG PS) of 0. In terms of comorbidities, he had hypothyroidism, hepatitis B, hypertension, and hyperuricemia; there was no history of drug allergies. The concomitantly used drugs were amlodipine, dextromethorphan, entecavir, febuxostat, levothyroxine, teprenone, loxoprofen, and rebamipide. Arthralgia appeared from around day 4 of sorafenib administration, followed by an intermittent fever of $39^{\circ} \mathrm{C}$. On day 11 , erythema appeared on the face and the anterior neck. On day 15, hand-foot syndrome was observed and erythema multiforme developed on the entire body, such as the face, both upper and lower limbs, and trunk (Figure 1A, B and C). Therefore, the patient was hospitalized to treat the suspected drug eruption. The clinical findings showed infiltrative erythema with a relatively clear boundary throughout the body and some target lesions. Furthermore, enanthem developed on the soft palate, and the patient experienced itching, mainly on the back. The reaction on the face was considered a severe acne-like skin rash; however, erythema multiforme could not be ruled out for the reaction on the trunk of the extremities. Blood culture, computed tomography, and urinalysis showed no abnormalities. Based on these findings, the patient was clinically diagnosed with erythema multiforme. In addition, skin biopsy of the chest was performed, and thinned skin tissue and vacuolar degeneration were observed at the border of the epidermis and dermis (Figure 1D and E). Infiltration of inflammatory cells, mainly comprising lymphocytes, was observed around capillaries on the surface of the dermis into the epidermis, and individual cell necrosis was observed in the epidermis. The treatment course is shown in Figure 2A. On day 15, sorafenib was discontinued, and topical steroids (hydrocortisone butyrate and difluprednate) and olopatadine $10 \mathrm{mg}$ daily were started. In addition, loxoprofen and rebamipide were discontinued because acetaminophen was used to treat pain and fever. However, on day 16, the infiltrating erythema on the whole body showed a tendency to expand; therefore, oral prednisolone $(20 \mathrm{mg}$ daily, increased to $40 \mathrm{mg}$ daily from day 17) was initiated. By day 17 , the severity of erythema past its peak and started to regress; fever and malaise also alleviated. On day 19, oral enanthem also started to improve, and the dose of prednisolone was gradually reduced according to the course of the symptoms. On day 22, systemic lupus erythema almost disappeared, and hand-foot syndrome started to resolve (it eventually disappeared on day 24). The patient was discharged on day 25 . On day 29 , erythema multiforme was resolved, and oral prednisolone was discontinued. No recurrence of skin disorders was observed even after the administration of oral prednisolone ended. After that, he received temsirolimus and nivolumab as the seventh- and eighth-line therapies, respectively, until disease progression. After disease progression, treatment was switched to best supportive care in May 2019 (the patient died in July of the same year).

Case 2. A 42-year-old, $57.1 \mathrm{~kg}$ man underwent nephrectomy for right RCC (stage pT3a, N0, M1) in December 2016. Since lung metastases were detected in December 2016, he received pazopanib, nivolumab ( $3 \mathrm{mg} / \mathrm{kg}$, every 2 weeks), axitinib, and everolimus as the first-, second-, third-, and fourth-line therapies, respectively, until disease progression. No serious skin disorder was observed during treatment with pazopanib, nivolumab, axitinib, and everolimus before the start of sorafenib administration. After disease progression, treatment was switched to sorafenib ( $800 \mathrm{mg}$ daily) as the fifth-line therapy in July 2019. The interval between the last dose of nivolumab and the start of sorafenib treatment was 154 days. At this point, the patient was categorized as having intermediate risk according to the MSKCC risk criteria and was in a good general condition with an ECOG PS of 0. In terms of comorbidities, the patient had hypertension; there was no history of drug allergies. The concomitantly used drugs were olmesartan, oxycodone (for pain), and codeine (for cough). A painful rash appeared on the precordium, face, and scalp from approximately day 10 after administration of sorafenib. On day 12 , the patient experienced difficulty in walking due to the development of hand-foot syndrome. In addition, he had a fever of $39^{\circ} \mathrm{C}$ and was highly fatigued. Hence, he was hospitalized to treat the rash. Clinical findings showed multiple fusions of small round erythema on the face, 
A

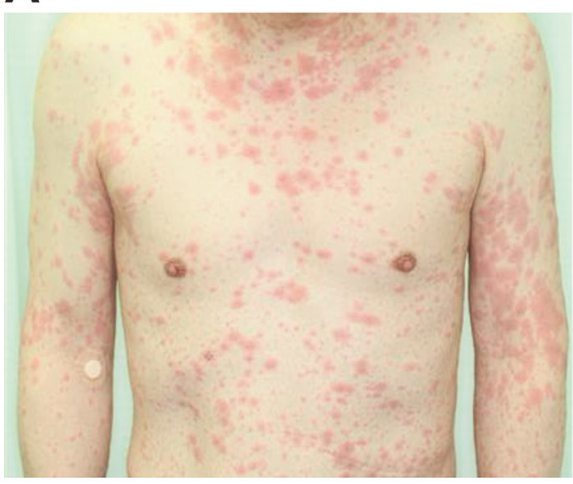

B

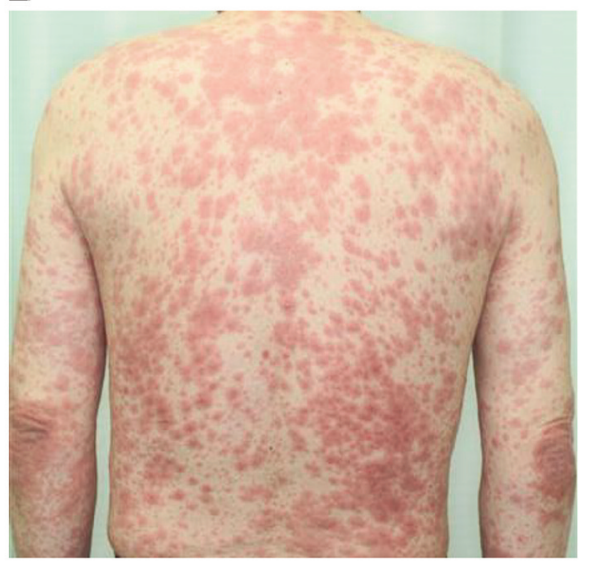

C

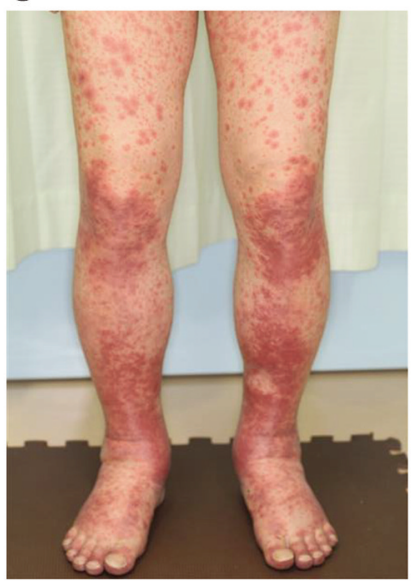

D

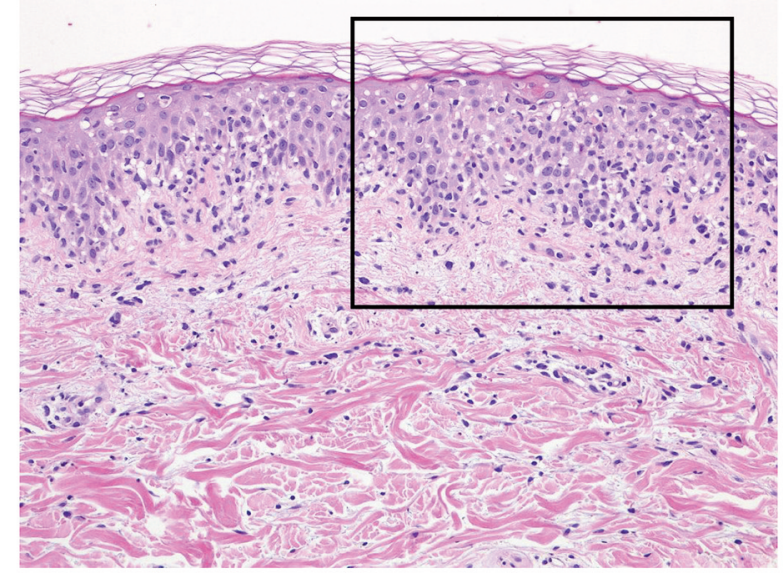

E

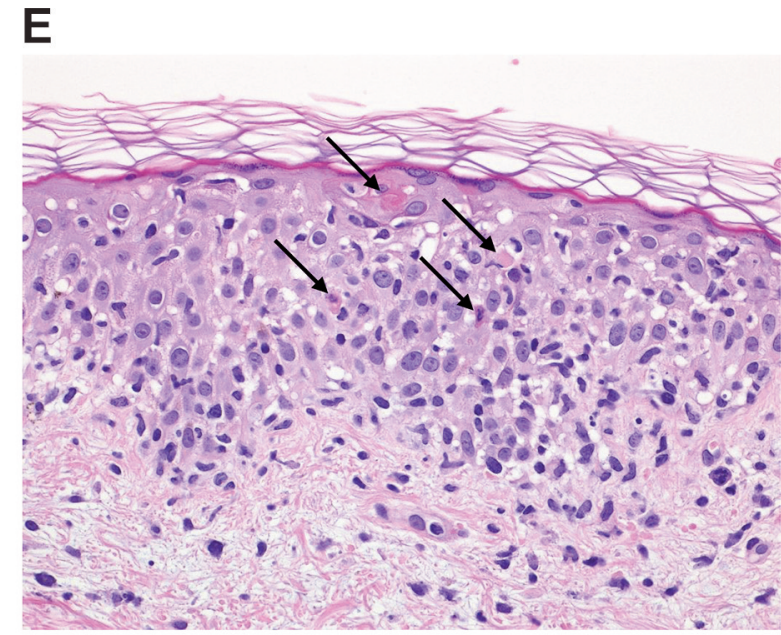

Figure 1. Infiltrative erythema seen in the front of body (A), back of body $(B)$, and the lower limbs $(C)$ on day 15 of sorafenib administration in Case 1 with severe erythema multiforme. Skin biopsy of the chest on day 15 of sorafenib administration in Case 1 . Hematoxylin and eosin staining (D, ×200; E, ×400). Arrow indicates individual cell necrosis.

and multiple solitary serous papules were found on the trunk, which were considered an acne-like skin rash (Figure 3A and B). Large blisters were scattered on the patient's fingers and toes, which were considered to be hand-foot syndrome (Figure 3C). Diffuse erythema, with desquamation in scarce zones, was found on the scalp. There were no findings suggestive of a bacterial infection. Tzanck test (cytodiagnosis of serous papules) showed inconspicuous eosinophils and bacteria, and nonspecific cell infiltration mainly by lymphocytes, and no giant viral cells. The treatment course is shown in Figure 2B. Sorafenib was discontinued on day 12 , and topical steroids (clobetasol propionate, a mixture of betamethasone valerate and gentamicin sulfate, and a mixture of clobetasol propionate and heparinoid), minocycline (200 mg daily until day 18), and fluid replacement rehydration (until day 15) were started. Along with sorafenib, olmesartan was prescribed for hypertension but was discontinued because the patient's blood pressure was within the normal range. On day 13 , the eruption began to spread, and on day 14, many round erythematous patches (1 $\mathrm{cm}$ in size) with relatively clear boundaries were noted on the face and trunk of the extremities. Furthermore, redness around the blisters on the first toe of both feet increased, and oral prednisolone $(15 \mathrm{mg}$ daily) was started. On day 16 , the patient's fatigue was relieved, and the rash did not spread. However, because he experienced itching, fexofenadine (120 mg daily until day 23) was started. Laboratory data showed that the inflammatory response was past its peak, which was considered to be an overall improvement trend. On day 17, the eruption also showed an improving tendency, and erythema on the trunk and upper limbs began to fade. In addition, the redness around the blisters on the first toe of both feet as well as the pain in the sole disappeared. The patient's fever also reduced. On day 18 , the eruption started to disappear due to the 


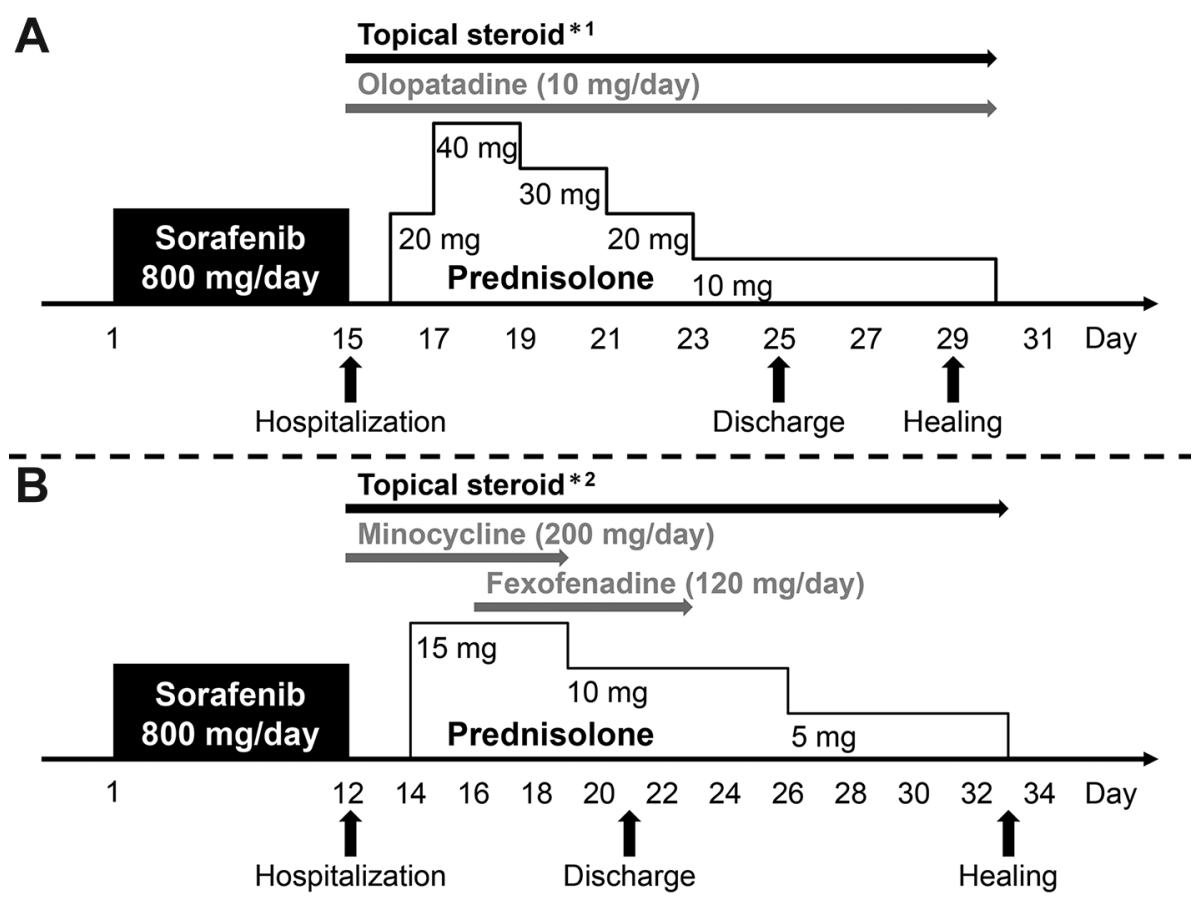

Figure 2. Treatment scheme in Case $1(A)$ and Case $2(B) . * 1:$ Hydrocortisone butyrate and difluprednate; $* 2$ : clobetasol propionate, mixture of betamethasone valerate and gentamicin sulfate, and mixture of clobetasol propionate and heparinoid.

A

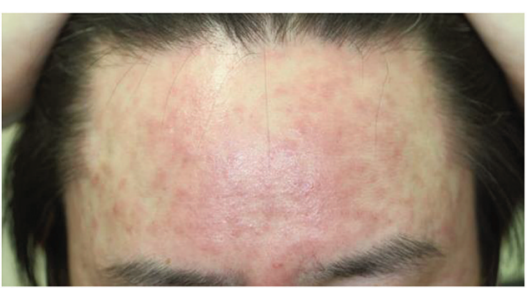

B

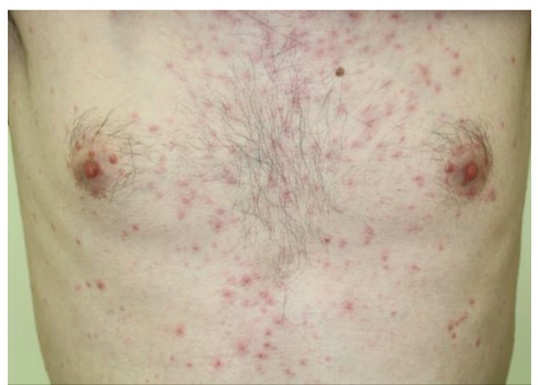

C

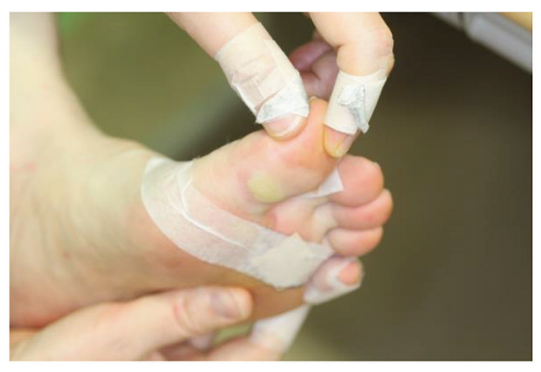

Figure 3. Multiple fusions of small round erythema on the face (A), multiple solitary serous papules on the trunk (B), and blisters on the first toe of left feet $(C)$ on day 12 of sorafenib administration of in the Case 2 with rash.

remarkable effect of steroids, and the redness of the face, limbs, and trunk began to disappear. Furthermore, the blisters on both soles shrank. On day 19, the eruption started to disappear, the inflammatory response improved, and the dose of prednisolone was gradually reduced according to the course of symptoms. Eventually, the patient was discharged on day 21. On day 33, the erythema was colorless and began to disappear; therefore, oral prednisolone was discontinued. No recurrence of skin disorders was observed thereafter. Subsequently, the patient received sunitinib and temsirolimus as sixth- and seventh-line therapies, respectively, until disease progression. After disease progression, the treatment was switched to sorafenib (400 mg daily) as the eighth-line therapy in July 2019 (the patient died in September of the same year). No skin disorder was observed after the readministration of sorafenib.

\section{Discussion}

Herein, we present two cases of severe skin disorders due to sorafenib use in patients with RCC who had been previously treated with nivolumab. The frequency of serious rash 
including erythema multiforme has been previously reported to be $6.7 \%$ in patients administered sorafenib monotherapy for unresectable or metastatic RCC (6). On the other hand, the frequency of grade 3 or 4 rash and erythema multiforme of any grade was $0.5 \%$ in patients who received nivolumab monotherapy for advanced RCC (7). As mentioned above, the frequency of severe skin disorders associated with sorafenib or nivolumab monotherapy is low. However, it cannot be ruled out that the severe skin disorders observed in these two cases may have developed because of the effects of sorafenib or nivolumab monotherapy. Currently, no data are available on the frequency of severe skin disorders caused by sorafenib in patients previously treated with nivolumab. The limited data available for the two patients suggest the possibility of severe skin rash caused by sorafenib after prior nivolumab treatment (8). In our institution, we have experienced only two cases of sorafenib use in patients previously treated with nivolumab; however, in both cases, the patients developed severe skin disorders as has been mentioned before. In contrast, a previous study reported that RCC patients who received tyrosine kinase inhibitors (pazopanib, sunitinib, axitinib, and cabozantinib) following ICIs developed no severe skin toxicity (9). These findings suggest that the severe skin disorder induced by tyrosine kinase inhibitor use following nivolumab treatment may be a characteristic adverse event associated with sorafenib. Therefore, further information is required on the safety of sorafenib use in patients previously treated with ICIs, such as nivolumab.

The mechanism of erythema multiforme is generally considered a type III or type IV allergy. In in situ and clinical studies (10), skin samples from patients with sorafenibinduced drug eruption, including erythema multiforme, showed an increase in the total number of mast cells, the number of degranulated mast cells, and the expression of stem cell factor, which plays an important role in the maturation and proliferation of mast cells, compared to that in healthy subjects. Furthermore, sorafenib was shown to promote the maturation and degranulation of skin-type mast cells. The promotion of skin-type mast cell maturation is due to the activation of the PI3K signaling pathway and increased stem cell factor production. These observations suggest that sorafenib could induce skin disorders by promoting the proliferation and degranulation of skin-type mast cells and infiltrating inflammatory cells such as CD8-positive T cells. On the other hand, nivolumab is known to be involved in the sensitization/activation or maintenance of CD8-positive $\mathrm{T}$ cell activation in the priming phase or effector phase (11), and a resultant immune overreaction might contribute to the development of serious skin disorders. In addition, PD-1 protein expression was noted in $33.3 \%$ of the cases of human cutaneous mastocytosis, and in vitro experiments revealed that the PD-1 signaling pathway negatively regulates stem cell factor-dependent proliferation of human mastocytosis
Table I. Summary of cases with severe skin disorders caused by sorafenib in patients previously treated with nivolumab

\begin{tabular}{|c|c|c|}
\hline & Case 1 & Case 2 \\
\hline Nivolumab dosage & $\begin{array}{c}3 \mathrm{mg} / \mathrm{kg} \text {, } \\
\text { every } 2 \text { weeks }\end{array}$ & $\begin{array}{c}3 \mathrm{mg} / \mathrm{kg} \text {, } \\
\text { every } 2 \text { weeks }\end{array}$ \\
\hline $\begin{array}{l}\text { Total cycles of } \\
\text { nivolumab administration }\end{array}$ & 10 cycles & 22 cycles \\
\hline $\begin{array}{l}\text { Time interval between } \\
\text { nivolumab and sorafenib }\end{array}$ & 28 days & 154 days \\
\hline Sorafenib dosage & $800 \mathrm{mg}$, daily & $800 \mathrm{mg}$, daily \\
\hline $\begin{array}{l}\text { Time to hospitalization } \\
\text { due to the onset of } \\
\text { serious skin disorders }\end{array}$ & 14 days & 11 days \\
\hline Skin toxicity & $\begin{array}{l}\text { Erythema multiforme, } \\
\text { acne-like skin rash, } \\
\text { hand-foot syndrome }\end{array}$ & $\begin{array}{l}\text { Acne-like skin rash, } \\
\text { hand-foot syndrome }\end{array}$ \\
\hline Treatment & $\begin{array}{l}\text { Steroids (topical } \\
\text { and oral) }\end{array}$ & $\begin{array}{l}\text { Steroids (topical } \\
\text { and oral) }\end{array}$ \\
\hline $\begin{array}{l}\text { Re-administrarion } \\
\text { of sorafenib }\end{array}$ & no & yes \\
\hline
\end{tabular}

cells (12). Therefore, nivolumab may also be directly involved in the proliferation of skin-type mast cells. Thus, sorafenib and nivolumab may work together to cause aberrant immune modulation and severe skin disorders.

The half-life of nivolumab in serum is approximately 17 days. However, nivolumab has been reported to bind to $\mathrm{T}$ cells more than 20 weeks after its last administration in patients with non-small cell lung cancer (1). In Case 1, erythema multiforme and pimple-like eruption were observed, and in Case 2, pimple-like eruption was observed (Table I). Considering the severity of the skin disorders in both cases, shorter dosing intervals between the final dose of nivolumab and the start of sorafenib treatment may lead to more serious skin disorders. Interestingly, in Case 2, no skin damage was observed due to the re-administration of sorafenib (eighth-line therapy), probably because of the extended interval from the last dose of nivolumab or because of the administration of betamethasone ( $4 \mathrm{mg}$ daily, injection, until 5 days after the re-administration of sorafenib) for fatigue.

\section{Conclusion}

Side effects may occur several months after the end of nivolumab administration; therefore, attention should be paid to the development of serious skin disorders due to sorafenib use in patients previously treated with nivolumab.

\section{Conflicts of Interest}

The Authors have declared no potential conflicts of interest in relation to this study. 


\section{Authors' Contributions}

Conceptualization: HK, SN, TY, NF, TT; Data curation: HK; Investigation: HK, SN, TY, SK, KT, TK, AK, NF; Project administration: TT; Resources: TY, SK, KT, TK, AK, NF; Supervision: TT; Visualization: HK, SN, KT, TK, NF; Writing original draft: HK, SN; Writing - review \& editing: TY, SK, KT, $\mathrm{TK}, \mathrm{AK}, \mathrm{NF}$.

\section{References}

1 Osa A, Uenami T, Koyama S, Fujimoto K, Okuzaki D, Takimoto T, Hirata H, Yano Y, Yokota S, Kinehara Y, Naito Y, Otsuka T, Kanazu M, Kuroyama M, Hamaguchi M, Koba T, Futami Y, Ishijima M, Suga Y, Akazawa Y, Machiyama H, Iwahori K, Takamatsu H, Nagatomo I, Takeda Y, Kida H, Akbay EA, Hammerman PS, Wong KK, Dranoff G, Mori M, Kijima T and Kumanogoh A: Clinical implications of monitoring nivolumab immunokinetics in non-small cell lung cancer patients. JCI Insight 3(19): e59125, 2018. PMID: 30282824. DOI: 10.1172/ jci.insight. 59125

2 Mamesaya N, Kenmotsu H, Katsumata M, Nakajima T, Endo M and Takahashi T: Osimertinib-induced interstitial lung disease after treatment with anti-PD1 antibody. Invest New Drugs 35(1): 105-107, 2017. PMID: 27599705. DOI: 10.1007/s10637-0160389-9

3 Takakuwa O, Oguri T, Uemura T, Sone K, Fukuda S, Okayama M, Kanemitsu Y, Ohkubo H, Takemura M, Ito Y, Maeno K and Niimi A: Osimertinib-induced interstitial lung disease in a patient with non-small cell lung cancer pretreated with nivolumab: A case report. Mol Clin Oncol 7(3): 383-385, 2017. PMID: 28808573. DOI: $10.3892 / \mathrm{mco} .2017 .1349$

4 Harding JJ, Pulitzer $M$ and Chapman PB: Vemurafenib sensitivity skin reaction after ipilimumab. N Engl J Med 366(9): 866-868, 2012. PMID: 22375995. DOI: 10.1056/NEJMc1114329

5 Johnson DB, Wallender EK, Cohen DN, Likhari SS, Zwerner JP, Powers JG, Shinn L, Kelley MC, Joseph RW and Sosman JA: Severe cutaneous and neurologic toxicity in melanoma patients during vemurafenib administration following anti-PD-1 therapy. Cancer Immunol Res 1(6): 373-377, 2013. PMID: 24490176. DOI: 10.1158/2326-6066.CIR-13-0092

6 Akaza H, Oya M, Iijima M, Hyodo I, Gemma A, Itoh H, Adachi M, Okayama Y, Sunaya T and Inuyama L: A large-scale prospective registration study of the safety and efficacy of sorafenib tosylate in unresectable or metastatic renal cell carcinoma in Japan: results of over 3200 consecutive cases in post-marketing all-patient surveillance. Jpn J Clin Oncol 45(10): 953-962, 2015. PMID: 26206897. DOI: 10.1093/jjco/hyv099
7 Motzer RJ, Escudier B, McDermott DF, George S, Hammers HJ, Srinivas S, Tykodi SS, Sosman JA, Procopio G, Plimack ER, Castellano D, Choueiri TK, Gurney H, Donskov F, Bono P, Wagstaff J, Gauler TC, Ueda T, Tomita Y, Schutz FA, Kollmannsberger C, Larkin J, Ravaud A, Simon JS, Xu LA, Waxman IM, Sharma P and CheckMate 025 Investigators: Nivolumab versus everolimus in advanced renal-cell carcinoma. N Engl J Med 373(19): 1803-1813, 2015. PMID: 26406148. DOI: $10.1056 /$ NEJMoa1510665

8 Verret B, Badoual C, Combe P, Carlotti A and Oudard S: Targeted therapy's skin toxicities after immunotherapy: Skin toxicity of tyrosine kinase inhibitor therapy following nilovumab therapy for metastatic renal cell carcinoma. Clin Oncol 1: 1153, 2016.

9 Shah AY, Kotecha RR, Lemke EA, Chandramohan A, Chaim JL, Msaouel P, Xiao L, Gao J, Campbell MT, Zurita AJ, Wang J, Corn PG, Jonasch E, Motzer RJ, Sharma P, Voss MH and Tannir NM: Outcomes of patients with metastatic clear-cell renal cell carcinoma treated with second-line VEGFR-TKI after first-line immune checkpoint inhibitors. Eur J Cancer 114: 67-75, 2019. PMID: 31075726. DOI: 10.1016/j.ejca.2019.04.003

10 Mizukami Y, Sugawara K, Kira Y and Tsuruta D: Sorafenib stimulates human skin type mast cell degranulation and maturation. J Dermatol Sci 88(3): 308-319, 2017. PMID: 28843624. DOI: $10.1016 /$ j.jdermsci.2017.08.005

11 Chen DS and Mellman I: Oncology meets immunology: the cancer-immunity cycle. Immunity 39(1): 1-10, 2013. PMID: 23890059. DOI: 10.1016/j.immuni.2013.07.012

12 Kataoka TR, Fujimoto M, Moriyoshi K, Koyanagi I, Ueshima C, Kono F, Tsuruyama T, Okayama Y, Ra C and Haga H: PD-1 regulates the growth of human mastocytosis cells. Allergol Int 62(1): 99-104, 2013. PMID: 23267208. DOI: 10.2332/allergolint.12-OA0450
Received May 27, 2021

Revised June 19, 2021

Accepted June 22, 2021 